\title{
A QFT-induced phase in neutrino flavour oscillations
}

\author{
Dharam Vir Ahluwalia ${ }^{a, b, *}$ \\ a Department of Physics, Indian Institute of Technology Guwahati, \\ Guwahati - 781 039, Assam, India and \\ ${ }^{b}$ Centre for the Studies of the Glass Bead Game \\ Chaugon, Bir, Himachal Pradesh 176 077, India \\ Cheng-Yang Lee† \\ Manipal Centre for Natural Sciences, Manipal University, \\ Manipal, Karnataka 576104, India
}

(Dated: 24 May 2017)

\begin{abstract}
In the extended Standard Model of particle physics, each neutrino mass eigenstate is predicted to have a tiny but non vanishing magnetic moment induced by quantum field theoretic corrections. These QFT-induced magnetic momenta depend linearly on masses of the underlying mass eigenstates with a proportionality constant $3 e G_{F} /\left(8 \sqrt{2} \pi^{2}\right)$. As a consequence when neutrinos are embedded in an environment containing magnetic fields the flavour oscillations get a contribution from the induced relative phases.
\end{abstract}

* d.v.ahluwalia@iitg.ac.in

$\dagger$ cy.lee@manipal.edu 
If Pontecorvo formalism to understand neutrino oscillations is correct, then neutrinos provide concrete evidence for physics beyond the Standard Model (SM). The observations of flavour oscillations then mean that neutrinos produced through electroweak processes must be in linear superposition of at least two non-vanishing masses contrary to the prediction of the SM - the lowest mass eigenstate may be massless, or carry a non-zero mass.

Due to quantum field theoretic corrections (QFT), various extensions of the SM predict that neutrinos should have small but non-zero magnetic moments [1-10]. For the Dirac neutrinos, the magnetic moments corresponding to the individual mass eigenstates are given by

$$
\begin{aligned}
\mu_{i} & =\frac{3 e G_{F} m_{i}}{8 \sqrt{2} \pi^{2}} \\
& =1.85 \times 10^{-27}\left(\frac{m_{i}}{\mathrm{eV}}\right)\left(\frac{\mathrm{eV}}{\text { Gauss }}\right)
\end{aligned}
$$

The physics of the neutrino magnetic moment have been extensively studied in the forms of spin precession in magnetic fields and the helicity-flipping scattering amplitudes [11 22$]$. In this letter, we report a new type of $\mu$-induced flavour oscillation that to the best of our knowledge, has not been considered. The crucial observation lies in the fact that since neutrinos are predicted to have non-zero magnetic moments, the energies, and hence the induced phases, of the mass eigenstates are shifted when travelling through magnetic fields. Moreover, this shift varies with the mass of the mass eigenstate in the superposition that defines the neutrino flavour. This circumstance adds a new element to amplitudes, and hence probabilities, for neutrino flavour oscillations.

A simple analysis shows that the kinematically induced phase

$$
\varphi_{j i} \equiv \frac{\Delta m_{j i}^{2} L}{4 E}=1.27\left(\frac{\Delta m_{j i}^{2}}{\mathrm{eV}^{2}}\right)\left(\frac{L}{\mathrm{~km}}\right)\left(\frac{\mathrm{GeV}}{E}\right)
$$

gets an additive QFT-induced correction

$$
\begin{aligned}
\varphi_{j i}^{\mathrm{new}} & \equiv \varphi_{j i}\left(\frac{3 e G_{F} B}{4 \sqrt{2} \pi^{2}}\right) \cos (\eta) \\
& =\varphi_{j i} \times 3.7 \times 10^{-27} \cos (\eta)\left(\frac{B}{\text { Gauss }}\right) .
\end{aligned}
$$

Where $\eta$ is the angle between the magnetic field (assumed constant and uniform) and the direction of propagation, and $\Delta m_{j i}^{2}=m_{j}^{2}-m_{i}^{2}$ is the usual mass-squared difference between 
the $i$ th and $j$ th mass eigenstates.

Some details of the analysis - The just quoted results require a calculation of the interaction energy of the magnetic moment of a Dirac mass eigenstate with an external magnetic field. We do this calculation for a uniform and constant magnetic field. The effective coupling of the neutrino to the classical electromagnetic field due to the magnetic moment is

$$
V=\int d^{3} x J_{\mathrm{eff}}^{\mu} A_{\mu}
$$

where $J_{\text {eff }}^{\mu}(x)$ is the current. We take its matrix elements to be of the form [19]

$$
\begin{aligned}
\left\langle p^{\prime}, \sigma^{\prime}\left|J_{\mathrm{eff}}^{\mu}(x)\right| p, \sigma\right\rangle & =e^{-i\left(p-p^{\prime}\right) \cdot x}\left\langle p^{\prime}, \sigma^{\prime}\left|J_{\mathrm{eff}}^{\mu}(0)\right| p, \sigma\right\rangle \\
& \equiv \frac{1}{2(2 \pi)^{3} \sqrt{E E^{\prime}}} e^{-i\left(p-p^{\prime}\right) \cdot x} \bar{u}\left(\mathbf{p}^{\prime}, \sigma^{\prime}\right) \Gamma^{\mu}\left(p^{\prime}, p\right) u(\mathbf{p}, \sigma)
\end{aligned}
$$

where $\Gamma^{\mu}\left(p^{\prime}, p\right) \equiv-i \mu \sigma^{\mu \nu}\left(p-p^{\prime}\right)_{\nu}$ with $\mu$ being the neutrino magnetic moment and $\sigma^{\mu \nu}=$ $(i / 2)\left[\gamma^{\mu}, \gamma^{\nu}\right]$, and the remaining symbols have their usual meaning. The normalization factor comes from our choice of expansion for the fermionic field

$$
\psi(x)=(2 \pi)^{-3 / 2} \int \frac{d^{3} p}{\sqrt{2 E}} \sum_{\sigma}\left[e^{-i p \cdot x} u(\mathbf{p}, \sigma) a(\mathbf{p}, \sigma)+e^{i p \cdot x} v(\mathbf{p}, \sigma) b^{\dagger}(\mathbf{p}, \sigma)\right] .
$$

Since we are interested in neutrinos propagating through a constant magnetic field, the potential is time-independent $A^{\mu}=A^{\mu}(\mathbf{x})$. We work in the temporal gauge and set $A^{0}(x)=$ 0 . With these facts noted, we obtain

$$
\left\langle p^{\prime}, \sigma^{\prime}|V| p, \sigma\right\rangle=-\frac{\mu}{E} \bar{u}\left(\mathbf{p}^{\prime}, \sigma^{\prime}\right) J^{i j} u(\mathbf{p}, \sigma) F_{j i} \delta^{3}\left(\mathbf{p}^{\prime}-\mathbf{p}\right)
$$

where $J^{i j}=(i / 4)\left[\gamma^{i}, \gamma^{j}\right]$ and $F_{i j}=\partial_{i} A_{j}-\partial_{j} A_{i}$. Using the identity $F_{i j}=-\epsilon_{i j k} B^{k}$, equation (7) simplifies to

$$
\left\langle p^{\prime}, \sigma^{\prime}|V| p, \sigma\right\rangle=-\left(\frac{\mu}{E}\right) \bar{u}\left(\mathbf{p}^{\prime}, \sigma^{\prime}\right)(\mathbf{J} \cdot \mathbf{B}) u(\mathbf{p}, \sigma) \delta^{3}\left(\mathbf{p}^{\prime}-\mathbf{p}\right) .
$$

Generally, the neutrinos produced in the weak interactions are relativistic helicity eigenstates. In this case, we have

$$
\bar{u}(\mathbf{p}, \sigma)(\mathbf{J} \cdot \mathbf{B}) u(\mathbf{p}, \sigma) \approx 2 m \sigma \widehat{\mathbf{p}} \cdot \mathbf{B}
$$

Therefore, by taking $p=p^{\prime}, \sigma=\sigma^{\prime}$ and normalising the matrix element, the shift in energy is given by

$$
V_{\mathrm{rel}} \equiv \frac{\left\langle p, \sigma\left|V_{\mathrm{rel}}\right| p, \sigma\right\rangle}{\langle p, \sigma \mid p, \sigma\rangle} \approx 2 \sigma\left(\frac{m}{E}\right)(\sim \widehat{\mathbf{p}} \cdot \mathbf{B}) .
$$


For completeness, in the non-relativistic limit where $\mathbf{p} \sim \mathbf{0}$, we have

$$
\bar{u}(\mathbf{0}, \sigma)(\mathbf{J} \cdot \mathbf{B}) u(\mathbf{0}, \sigma)=2 m \sigma B_{z}
$$

SO

$$
V_{\text {non-rel }} \equiv \frac{\left\langle k, \sigma\left|V_{\text {non-rel }}\right| k, \sigma\right\rangle}{\langle k, \sigma \mid k, \sigma\rangle} \approx-2 \sigma \mu B_{z}
$$

where $k^{\mu}=(m, \mathbf{0})$ and $B_{z}$ is the magnetic field along the $z$-axis. The spin of the state $|k, \sigma\rangle$ is aligned with the $z$-axis.

Concluding remarks - A class of neutron stars called magnetar can have magnetic fields of the order of $10^{16}$ Gauss [23]. This falls some eleven orders of magnitude too short to effect neutrino oscillations in the present cosmic epoch. However, with cosmic expansion the magnetic field scales as $B_{e}=B_{p}\left(a_{p} / a_{e}\right)^{2}$ where $a$ is the scale factor, while $e$ refers to the end of inflation and $p$ denotes the present epoch. Trivedi and Subramanian argue that CMB observations provide an upper limit for $B_{p}$ to be $\lesssim 0.05$ nano-Gauss [24]. It is not clear to us as to how far back we may extrapolate the naive increase in the magnetic field to early universe to entertain a dramatic change in neutrino oscillations.

Acknowledgement - C. Y. L would like to thank the generous hospitality offered by the Department of Physics and Astronomy of the University of Canterbury where part of this work was completed.

[1] W. J. Marciano and A. I. Sanda, Exotic decays of the muon and heavy leptons in gauge theories, Phys. Lett. B67 (1977) 303-305.

[2] B. W. Lee and R. E. Shrock, Natural suppression of symmetry violation in gauge theories: Muon - lepton and electron lepton number nonconservation, Phys. Rev. D16 (1977) 1444.

[3] P. B. Pal and L. Wolfenstein, Radiative decays of massive neutrinos, Phys. Rev. D25 (1982) 766.

[4] J. Schechter and J. W. F. Valle, Majorana neutrinos and magnetic fields, Phys. Rev. D24 (1981) 1883-1889. [Erratum: Phys. Rev.D25,283(1982)].

[5] R. E. Shrock, Electromagnetic properties and decays of Dirac and Majorana neutrinos in a general class of gauge theories, Nucl. Phys. B206 (1982) 359-379.

[6] S. S. Masood, Neutrino physics in hot and dense media, Phys. Rev. D48 (1993) 3250-3258. 
[7] S. S. Masood, Magnetic moment of neutrinos in the statistical background, Astropart. Phys. 4 (1995) 189-194.

[8] M. S. Dvornikov and A. I. Studenikin, Electromagnetic form-factors of a massive neutrino, J. Exp. Theor. Phys. 99 (2004) 254-269, hep-ph/0411085.

[9] N. F. Bell, V. Cirigliano, M. J. Ramsey-Musolf, P. Vogel, and M. B. Wise, How magnetic is the Dirac neutrino?, Phys. Rev. Lett. 95 (2005) 151802, hep-ph/0504134.

[10] N. F. Bell, How magnetic is the neutrino?, Int. J. Mod. Phys. A22 (2007) 4891-4899, arXiv:0707.1556.

[11] A. Cisneros, Effect of neutrino magnetic moment on solar neutrino observations, Astrophys. Space Sci. 10 (1971) 87-92.

[12] K. Fujikawa and R. E. Shrock, The magnetic moment of a massive neutrino and neutrino spin rotation, Phys. Rev. Lett. 45 (1980) 963.

[13] M. S. Dvornikov and A. I. Studenikin, Neutrino spin evolution in presence of general external fields, JHEP 09 (2002) 016, hep-ph/0202113.

[14] A. I. Studenikin, The four new effects in neutrino oscillations, Nucl. Phys. Proc. Suppl. 143 (2005) 570-570, hep-ph/0407010.

[15] A. I. Studenikin, Neutrinos in electromagnetic fields and moving media, Phys. Atom. Nucl. 67 (2004) 993-1002. [Yad. Fiz.67,1014(2004)].

[16] C. Giunti and A. I. Studenikin, Neutrino electromagnetic properties, Phys. Atom. Nucl. 72 (2009) 2089-2125, arXiv:0812.3646].

[17] C. Giunti and A. I. Studenikin, Electromagnetic properties of neutrinos, J. Phys. Conf. Ser. 203 (2010) 012100, arXiv:1006.1502].

[18] C. Broggini, C. Giunti, and A. I. Studenikin, Electromagnetic Properties of Neutrinos, Adv. High Energy Phys. 2012 (2012) 459526, [arXiv:1207.3980].

[19] C. Giunti and A. I. Studenikin, Neutrino electromagnetic interactions: a window to new physics, Rev. Mod. Phys. 87 (2015) 531, arXiv:1403.6344].

[20] A. Dobrynina, A. Kartavtsev, and G. Raffelt, Helicity oscillations of Dirac and Majorana neutrinos, Phys. Rev. D93 (2016), no. 12 125030, [arXiv:1605.0451].

[21] R. Fabbricatore, A. Grigoriev, and A. I. Studenikin, Neutrino spin-flavor oscillations derived from the mass basis, J. Phys. Conf. Ser. 718 (2016), no. 6 062058, [arXiv:1604.0124].

[22] A. I. Studenikin, Status and perspectives of neutrino magnetic moments, in 14 th 
International Conference on Topics in Astroparticle and Underground Physics (TAUP 2015) Torino, Italy, September 7-11, 2015, 2016. arXiv:1603.0033.

[23] A. Tiengo et al., A variable absorption feature in the X-ray spectrum of a magnetar, Nature 500 (2013) 312, arXiv:1308.4987].

[24] P. Trivedi, K. Subramanian, and T. R. Seshadri, Primordial magnetic field limits from the CMB trispectrum: Scalar modes and Planck constraints, Phys. Rev. D 89 (Feb, 2014) 043523. 